\title{
PERCEPÇÃO DE BEM-ESTAR ENTRE FUNCIONÁRIOS ATIVOS E DEMISSIONÁRIOS DE UMA ORGANIZAÇÃO DA SAÚDE NA REGIÃO METROPOLITANA DA GRANDE VITÓRIA
}

PERCEPTION OF WELL-BEING BETWEEN ACTIVE AND DEMISSIBLE EMPLOYEES OF A HEALTH ORGANIZATION IN THE METROPOLITAN REGION OF THE GREAT VICTORY

\section{Katiana Erler}

Mestre em Administração, Fucape Business School.

Data de recebimento: 03/07/2017

Marcia Juliana d'Angelo

Data de aceite: 05/12/2017

Doutora em Administração de Empresas, Fucape Business School.

\section{RESUMO}

Esta pesquisa quantitativa compara a percepção de bem-estar no trabalho entre funcionários ativos e demissionários de uma organização do terceiro setor no segmento da saúde, na Região Metropolitana da Grande Vitória, a partir de fatores pessoais e situacionais. A composição da amostra final foi de 265 questionários válidos, sendo 132 de funcionários ativos e 133 de profissionais que solicitaram demissão voluntária. Por meio da análise de Teste t, os resultados mostram que há diferenças significativas entre esses dois grupos de funcionário, no tocante à realização no trabalho, mas não tocante aos afetos positivos e negativos. Chama a atenção que os funcionários que solicitaram a demissão voluntária se sentiram mais dispostos e animados nesse ambiente de trabalho do que os ativos. Quanto aos sentimentos negativos, os funcionários percebem ou perceberam só um pouco tais emoções, não havendo diferenças significativas entre os dois grupos. E, para ambos os grupos, ganhar recompensas importantes é pouco percebida.

Palavras-chave: Bem-estar no Trabalho. Rotatividade de Pessoal. Gestão de Pessoas.

\section{ABSTRACT}

This research compares the perception of well-being at work between active and resigning employees from a third sector organization in the health sector, in the metropolitan area of Vitoria, from personal and situational factors. The final sample consisted of 265 valid questionnaires, 132 from active employees and 133 from resigning employees. Through the analysis of Test $t$, the results show that there are significant differences between these two groups of employees, regarding the accomplishment at work, but not concerning the positive and negative affects. It draws attention to employees who requested the resignation felt more willing and excited in this work environment than the active ones. As for the negative feelings, employees perceive or perceived just a little such emotions and there is no difference between the two groups. In addition, both groups perceive they do not gain important rewards.

Keywords: Well-being at Work. Turnover. People management.

Endereço dos autores:

Katiana Erler

pesquisa@fucape.br
Marcia Juliana d'Angelo

marciadangelo@fucape.br 


\section{INTRODUÇÃO}

Para se manterem competitivas, as organizações buscam continuamente a valorização dos seus funcionários (AGAPITO et al., 2015) que, por sua vez, veem o trabalho como parte de uma trajetória de construção de relacionamentos e experiências, além de sobrevivência (DUFFY et al., 2015). Nesse contexto, a concepção de que a felicidade pode ser alcançada por meio do trabalho impulsiona as organizações a buscarem estratégias que promovam o bem-estar dentro do ambiente de trabalho (MIHAIL; KLOUTSINIOTIS, 2016; SANT'ANNA; PASCHOAL; GOSENDO, 2012). Bem-estar no trabalho está relacionado às emoções positivas e à tomada de consciência do funcionário de que é possível se realizar ao longo de uma trajetória laboral (PASCHOAL; TAMAYO, 2008). Aliás, felicidade é uma forma intercambiável de bem-estar (HEFFERNAN; DUNDON, 2016; VAN DE VOORDE et al., 2012; PASCHOAL et al., 2010; FISHER, 2010; ASHKANASY, 2009).

Juntos, esses fatores podem contribuir para minimizar a rotatividade dos funcionários nas empresas (AGAPITO et al., 2015; SIU et al., 2015), que se refere ao fluxo de entrada e saída entre a força de trabalho (ASSIS, 2012). Desta forma, há organizações preocupadas em proporcionar aos seus funcionários condições necessárias como um ambiente de trabalho mais autêntico para que alcancem boa performance, um grau de satisfação elevado (VAN DEN BOSCH; TARIS, 2014; REGO; CUNHA, 2012) e desenvolvam uma carreira bem-sucedida (RAHIM, 2017; REGO, 2009). Estudos mostram que cuidar do bem-estar tem efeitos positivos na produtividade, absenteísmo e comprometimento afetivo dos funcionários (SHIRRMEISTER; FRANÇA, 2012).

De maneira geral, a demissão voluntária de funcionários vem impulsionando um aumento das pesquisas que identifiquem fatores que contribuam para a retenção dos funcionários nas empresas (LEE et al., 2012; CAMPOS; MALIK, 2008). Embora haja várias pesquisas para discutir bem-estar no trabalho (BRAGA et al., 2017; PASCHOAL; TAMAYO, 2008; SIQUEIRA; PADOVAM, 2008;) e rotatividade (AGAPITO et al., 2015), estas foram realizadas em uma única organização, dificultando a generalização dos resultados. Daí a relevância em conduzir mais estudos em outros segmentos do mercado, bem como em outras cidades e regiões (SANCHO et al., 2011). Além disso, a replicação sistemática de um estudo utilizando diferentes amostras (com grupos diferentes, por exemplo, funcionários ativos e demissionários) e medições contribui para generalizar os resultados e para reduzir suas limitações (BEHSON, 2005), já que generalizações a partir de um único estudo são limitadas (BENSON; BROWN, 2011).

Diante desse contexto, este estudo apresenta o seguinte problema de pesquisa: como fatores situacionais e pessoais impactam o bem-estar no trabalho de profissionais ativos e de profissionais que decidiram pela demissão voluntária? Assim, este estudo objetiva comparar os impactos sobre o bem-estar no trabalho dos profissionais ativos e de profissionais que decidiram pela demissão voluntária de uma organização de grande porte no segmento da saúde na Grande Vitória.

\section{REFERENCIAL TEÓRICO}

\subsection{FELICIDADE E BEM-ESTAR - CONCEITOS INTERCAMBIÁVEIS}

O termo felicidade é muito usado pelo senso comum. Por exemplo, o Dicionário Houaiss da 
Língua Portuguesa (2004, p. 67) define felicidade como: (i) "qualidade ou estado de feliz, estado de uma consciência plenamente satisfeita, satisfação, contentamento, bem-estar"; (ii) "boa fortuna, sorte"; e (iii) "bom êxito, acerto, sucesso". Assim, entende-se como um estado de satisfação, contentamento, ou seja, emoções positivas. Trata-se de um conceito amplo e genérico, construído por emoções transmitidas em forma de alegria, referindo-se a um estado emocional de satisfação, ou seja, de uma forma intercambiável de bem-estar (FISHER, 2010; ASHKANASY, 2009).

Em geral, indivíduos felizes são mais propensos a terem casamentos e relações estáveis, rendimentos profissionais elevados, maior interação com a comunidade em que estão inseridos; além de boa saúde e vida longa. Cabe dizer que a felicidade fomenta a construção e compartilhamento de habilidades e conhecimentos (LYUBOMIRSKY et al., 2005). Por isso, as organizações poderiam proporcionar um ambiente com clima saudável aos seus integrantes, de modo que eles possam exercer sua criatividade e, consequentemente, usufruir o direito inalienável de serem felizes (REGO, 2009). Em troca, a organização tenderá a receber índices elevados de produtividade no longo prazo (SHIRRMEISTER; LIMONGI-FRANÇA, 2012; TORRISI, 2013).

Adicionalmente, felicidade é considerada uma das três dimensões para discutir bem-estar e diz respeito à satisfação no trabalho e compromisso com a organização. A segunda dimensão refere-se à saúde com foco nos fatores que geram o estresse e a terceira dimensão refere-se às formas de relacionamento de bem-estar (VAN DE VOORDE et al., 2012). Embora bem-estar possa ser discutido por meio do engajamento no trabalho, da Síndrome de
Burnout e da satisfação no trabalho (VAN DEN BOSCH; TARIS, 2014), neste estudo bem-estar remete ao bom funcionamento psicológico e às experiências positivas e negativas (SORAGGl; PASCHOAL, 2011). Abarca duas perspectivas, que diferem na definição do termo da felicidade: a hedonista, que associa o bem-estar com a presença de prazer e se intitula de bem-estar subjetivo; e a eudaimônica, que associa o bem-estar com o potencial humano e trata de bem-estar psicológico, que por sua vez “[...] apoia-se no escrúpulo de que bem-estar compreende-se no ápice do potencial possível de se alcançar de um individuo" (SIQUEIRA; PADOVAN, 2008, p. 201).

O Bem-Estar também pode ser desmembrado em duas dimensões: objetiva e subjetiva. A dimensão objetiva remete-se ao mensurável, palpável e que reflete nos níveis de padrões de vida do indivíduo, tais como: moradia, educação, emprego, situação financeira. Como, por exemplo, a satisfação com o chefe direto, colegas e remuneração. Já a dimensão subjetiva remete à forma como o ser humano percebe sua vida, a consonância entre seu ideal e sua realidade (CHEN et al., 2017). Ou seja, abrange as avaliações que os indivíduos fazem de suas vidas, a vivência pessoal de cada um; isto posto, compreende-se que cada pessoa avalia uma mesma situação de formas distintas, tendo em vista suas expectativas, valores etc. Como por exemplo, o feedback emocional remetido ao ambiente de trabalho, que pode ser notado nos sentimentos de desconforto, ansiedade, dentre outros (SIQUEIRA; PADOVAN, 2008). Diz respeito à três dimensões independentes e que podem ter causas diferentes: satisfação com a vida, experiências positivas e negativas. Todas têm correlações com a renda e 
aspirações das pessoas e como a renda é ganha e gasta; com as diferenças societais e culturais e a capacidade de adaptação das pessoas (DIENER, 1984; 2013). Ou à satisfação com a vida, com a saúde e afeto (LACHOWSKA, 2017), que mostrou que descontos fiscais impactam a redução do estresse e com as preocupaçoes, em geral, proporcionando bem-estar emocional, em particular, as pessoas de baixa renda.

Em particular, o bem-estar subjetivo, foco desta pesquisa divide-se em duas perspectivas em psicologia: afeto positivo e negativo, remetendo a estados de excitação, emoções, simpatia e sentimentos; os domínios de cognição, referindo-se ao conhecimento de contentamento com vida (SIQUEIRA; PADOVAN, 2008). Os afetos positivos referem-se a humores/emoções de alegria, de prazer ativo, de entusiasmo e de atividade. Já os afetos negativos referem-se a humores/emoções de distração, emoções desagradáveis, dentre outros.

No tocante às organizações, o conceito de bem-estar subjetivo, sob a perspectiva da psicologia positiva, está relacionado aos efeitos positivos da vivência do colaborador no trabalho. Ou seja, tem a ver com os efeitos que a satisfação com a vida, resiliência, espiritualidade, felicidade, dentre outros, impactam o bem-estar do funcionário na organização (PASCHOAL; TORRES; PORTO, 2010). Por se tratar de um "construto psicológico multidimensional" (NARDI; PALMA, 2015, p. 127), que envolve relações de sentimentos agradáveis e afetivos positivos tanto com o trabalho quanto com a organização, que poderia criar um ambiente que favoreça programas e ações de cunho interdisciplinar e transversal, contemplando uma visão holística do ser.
Particularmente, em hospitais, cuja "atividade laboral é caracterizada por extrema carga de trabalho, relacionamentos com situações de prazo, excesso de tensão e de riscos para outros ao redor e si próprio" (PASCHOALINI et al., 2008, p. 488; ELIAS; NAVARRO, 2006). Por isso, há necessidade de práticas de recursos humanos voltadas para a retenção e recrutamento de pessoal de saúde. Por exemplo, Fan et al. (2014) mostraram que no setor de saúde chinês, que quando a organização adota um modelo de gestão de alto desempenho com foco na motivação, habilidades e oportunidades há impacto positivo no bem-estar objetivo e no burnout. Já Mihail e Kloutsiniotis (2016) mostraram que um modelo de gestão com foco de alto desempenho com foco no recrutamento, treinamento, tomada de decisão, segurança do trabalho, autonomia do funcionário pode influenciar o esgotamento emocional ou o engajamento no trabalho, dependendo da percepção da troca social com o empregador.

\subsection{BEM-ESTAR E ROTATIVIDADE DA FORÇA DE TRABALHO}

Diversos estudos têm mostrado o impacto do bem-estar dos funcionários na intenção de sair da organização. Quando os funcionários percebem gatilhos de estresse específicos (monitoramento de desempenho, roteiros restritos, controle por meio de espaços físicos, câmeras, horas de trabalho, potencial para promoções) eles tendem a ter reações de sintomatologia somática (reações fisiológicas ao trabalho estressante), que por sua vez, impactam a intenção de sair de funcionários de call-center (MELLOR et al., 2015). Um estudo 
conduzido nos Estados Unidos, mostrou uma relação positiva entre a qualidade percebida da supervisão clínica, a percepção dos conselheiros quanto à autonomia do trabalho no tocante a dois elementos da teoria da justiça, o esgotamento emocional e à intenção de sair dos conselheiros da clínica (KNUDSEN et al., 2008).

Por outro lado, estratégias de intervenção voltadas para a reduzir a exaustão emocional, aumento de recursos têm impactos na satisfação do trabalho e na rotatividade de enfermeiras na Australia (RICKARD et al., 2012). As emoções positivas relacionadas ao capital psicológico, ou seja, a força do indivíduo baseada na autoconfiança, otimismo, esperança, resiliência melhora o bem-estar do trabalho e, por conseguinte, reduz a intenção dos policiais de Hong Kong a deixarem a organização (SIU et al., 2015). Já os profissionais de enfermagem em Portugal demonstraram que a busca pelo aprimoramento profissional e por atividades instigantes pode ajudar na explicação da rotatividade desses funcionários que, aparentemente, está em um nível aceitável para o setor. Os fatores de relação com contratos e remuneração também são relevantes na medida em que o funcionário se sente sensível devido à instabilidade contratual e à baixa remuneração, de acordo com (POEIRA; MAMEDE, 2011). Nomura e Gaidzinski (2005) mostraram que a rotatividade na equipe de enfermagem é baixa em um Hospital Universitário de São Paulo, porque tal organização provém ao funcionário oportunidades de conhecimento e habilidade técnicas, promoções e um clima de trabalho agradável, e estes fatores influenciam no bem-estar dos funcionários.

A demissão involuntária é preocupante pois essa atitude pode desenvolver na organiza- ção alguns problemas, como por exemplo, perda de conhecimento, redução temporária de produção, custos com processos trabalhistas e outros, portanto, o desafio está na contratação de profissionais, a partir de um planejamento estratégico, que se adeque às necessidades da organização para evitar futuros desligamentos. Desta forma, motivos à parte, a organização, ao controlar o índice de rotatividade, evita custos extras e desgastes dos funcionários, além do tempo destinado ao processo de recrutamento, seleção e treinamento. No setor de saúde, os níveis recomendados para a rotatividade são: até 1,75\%, esperado; até 3,75\%, aceitável; até 6,25\%, ruim; > 6,25\%, crítico (SANCHO et al., 2011).

\section{METODOLOGIA}

Para cumprir o objetivo, foi conduzido um estudo quantitativo com corte transversal porque os dados foram coletados em um ponto único na trajetória dos funcionários com o objetivo de extrair apenas uma amostra (MALHOTRA, 2006; HAIR JR. et al., 2005).

Para tanto, a população do estudo compreendeu os funcionários ativos (que se encontravam com vínculo empregatício ativo com a instituição) e os funcionários que decidiram pela demissão voluntária do setor privado. Já a amostra contemplou os profissionais ativos e que decidiram pela demissão voluntária de uma organização de grande porte no segmento da saúde localizada na Região Metropolitana da Grande Vitória. Essa organização possui como titulação Filantropia e Organização Social Saúde, atuando no mercado da saúde há mais de 40 anos. Atualmente possui uma 
unidade própria no município de Vila Velha, e gerencia duas unidades públicas, a saber: o Hospital Maternidade de Cariacica e Hospital Estadual Jayme Santos Neves, no município da Serra, totalizando a gestão média de 1.000 leitos de internação. É salutar informar que a pesquisa foi estudada em dois hospitais da organização AEBES, sendo elas, Hospital Estadual Jayme Santos Neves e Hospital Evangélico de Vila Velha. Tem chamado a atenção de estudos voltados para compreender a relação entre bem-estar e profissionais da área de saúde (GOETZ et al., 2015), que mostram fatores psicossociais podem afetar a saúde e os resultados no trabalho e, portanto, o bem-estar no trabalho.

O questionário escolhido para aplicação do bem-estar foi a Escala de Bem-Estar no Trabalho (EBET) de Paschoal e Tamayo (2008), por se tratar de uma escala com parâmetros psicométricos validados, ser de fácil aplicação em diferentes contextos e ambientes de trabalho. É composta por indicadores de emoções positivas (nove itens) e negativas (12 itens) que são medidos por meio de uma escala Likert de cinco pontos de 1 [nem um pouco] a 5 [extremamente]. Também compõem essa escala os indicadores de realização (nove itens) medidos também por meio de uma escala Likert de cinco pontos de 1 [discordo totalmente] a 5 [concordo totalmente]. Para complementar essa escala, foram solicitadas informações demográficas, como descrição do cargo, tempo de empresa, sexo, estado civil, filhos, faixa etária, renda familiar e escolaridade.

Para os profissionais ativos, o questionário foi aplicado pela própria pesquisadora, em horários de intervalos de refeições, nas imediações dos vestiários em diferentes horários e, também, no período de revezamento dos turnos. Para a po- pulação demissionária, no momento da entrega do questionário, era entregue outro questionário institucional da organização, denominado de "Entrevista de Desligamento". Esse instrumento é padronizado pela instituição para aplicação no momento de qualquer demissão para compreender os motivos pelos quais o funcionário tomou a decisão de solicitar a demissão da organização, tais como: acolhimento no setor de trabalho ao ser admitido; aprovação da atuação do seu gestor no período em que atuaram juntos; oferta de oportunidades para o seu crescimento profissional; e se o profissional voltaria a trabalhar na empresa, além de comentários adicionais. Essa etapa ocorrera em até 48 horas após solicitação da demissão. Trata-se, portanto, de amostras não probabilísticas, e por conveniência, porque o pesquisador utilizou critérios subjetivos, como a sua experiência pessoal e o seu conhecimento especializado com a organização para selecionar os respondentes mais disponíveis para tomar parte no estudo (HAIR JR. et al., 2005). A aplicação dos questionários para os profissionais ativos foi feita em Abril e Maio de 2014 e, para os profissionais solicitaram demissão voluntária do hospital, no período de Janeiro a Dezembro de 2014.

\subsection{TRATAMENTO ESTATÍSTICO DOS DADOS}

O questionário foi distribuído para uma amostra de 1.800 profissionais ativos que se encontravam com vínculo empregatício ativo na instituição, e para uma amostra de 155 funcionários que solicitaram demissão voluntária do hospital. Houve um retorno com respostas de 265 questionários válidos para a pesquisa, sendo 132 questionários da 
amostra de ativos e 133 questionários da amostra de profissionais que solicitaram demissão voluntária.

A preparação dos dados para análise seguiu as recomendações de Hair Jr. et al. (2005). Para tratar os dados faltantes e com o objetivo de não reduzir o tamanho da amostra, foi utilizada a abordagem da estimativa dos dados ausentes substituindo-se pela média. Em seguida, com o objetivo de se trabalhar com os mesmos fatores utilizados na Escala de Bem- Estar no Trabalho (EBET), de Paschoal e Tamayo (2008), os dados foram transformados por meio do cálculo dos escores somados para representar os fatores afeto positivo, afeto negativo e realização.

As suposições de normalidade foram verificadas por meio do teste estatístico de Kolmogorov-Smirnov, que mostrou resultados significativos $(0,000)$ ao nível de $p$ value $<0,05$ para todos os fatores, e complementadas por análises gráficas. As análises dos diagramas de dispersão para todos os fatores e respectivas variáveis não apresentaram relações não lineares aparentes. E as suposições de homocedasticidade foram avaliadas em conjunto com o teste das amostras independentes - Teste T para saber se há diferenças significativas na percepção de bem-estar entre os funcionários ativos e os que solicitaram demissão voluntária do hospital. E, para se conhecer e descrever o perfil dos respondentes dessa pesquisa, foram utilizadas estatísticas descritivas, como a média e o desvio-padrão.

\section{ANÁLISE DOS DADOS}

\subsection{DADOS DEMOGRÁFICOS DA AMOSTRA}

Nessa pesquisa, 49,8\% dos respondentes correspondem aos funcionários ativos, que ainda estão trabalhando no hospital, e 50,2\% referem-se aos profissionais que solicitaram demissão voluntária, que tomaram a decisão pela saída voluntária do hospital. Desse total, 89,8\% são do sexo feminino e apenas $10,2 \%$ do sexo masculino. Essa questão gênero é uma particularidade representativa do setor de saúde no Brasil, que predominantemente opera suas atividades com mulheres, sobretudo com profissionais de enfermagem (LOPES; LEAL, 2005). A maioria dos respondentes (82,9\%) ainda é muito jovem, estando na faixa etária entre 18 e 39 anos, o que demonstra existir a predominância nessa organização da geração Y, que vê o trabalho como satisfação, tendo como equilíbrio a qualidade de vida (VASCONCELOS, et al., 2010).

Quanto ao quesito escolaridade, 61,9\% dos respondentes tem pelo menos o ensino médio completo, consoante com o perfil dessa amostra composta por $90 \%$ de profissionais de enfermagem e médicos, uma exigência da legislação brasileira. Quanto à renda dos funcionários, 75\% recebem salários de até $R \$ 1.866,00$. No segmento da saúde, sobretudo nas categorias da profissão de enfermagem, existem variáveis diversas de estresse, e uma delas é a baixa remuneração (ELIAS; NAVAR$\mathrm{RO}, 2006)$.

Finalmente, também foi verificado que 77\% da amostra possui menos de dois anos de empresa, fato esse que evidencia o perfil presente de profissionais neófitos (pessoa recém-admitida em uma empresa). Segundo Siqueira e Gomide Jr. (2014), o índice de rotatividade da empresa é um indicativo de insatisfação no trabalho e se trata de fator relevante a ser avaliado, porque a conexão afetiva com a organização, positiva ou negativa, pode elevar o índice de rotatividade. 


\subsection{ESTATÍSTICA DESCRITIVA DOS PROFISSIONAIS ATIVOS}

De acordo com a Tabela 1, a média do fator realização no trabalho $(M=4,23)$ mostra evidências estatísticas de que os funcionários que trabalham nesse hospital concordam que conseguem se realizar no trabalho. A única exceção é que os profissionais só concordam em parte que conseguem recompensas importantes nesse trabalho $(M=3,71)$. A média do fator afeto positivo $(M=3,08)$ também mostra evidências estatísticas de que a afeição ao trabalho, isto é, o sentimento de emoções positivas, impacta de forma moderada o bem-estar dos funcionários desse hospital. Contudo, as médias para as variáveis orgulhoso $(\mathrm{M}=2,94)$, entusiasmo $(M=2,84)$, e empolgado $(M=2,77)$ ficaram abaixo do ponto médio, mostrando que os funcionários ativos sentem pouco tais emoções nesse ambiente de trabalho. E a média do fator afeto negativo ( $M$ $=2,04$ ) indica que as emoções negativas quanto ao trabalho impactam um pouco o bem-estar dos funcionários ativos desse hospital. As médias para as variáveis preocupado $(M=2,93)$ e ansioso $(M=$ $2,61)$ mostram que esses funcionários têm sentido mais essas emoções aflitivas.
O desvio padrão para todos os fatores realização $(D P=0,596)$, afeto positivo $(D P=0,860)$ e afeto negativo ( $D P=0,698)$ - está abaixo de 1,0, mostrando pouca variabilidade nas respostas, o que indica coerência nas respostas dos funcionários ativos desse hospital. Ou seja, aparentemente, há uma equivalência entre as situações e acontecimentos vivenciados por esses funcionários no tocante às emoções, negativas e positivas, e às oportunidades de realização nesse ambiente organizacional.

Contudo, ao analisar cada variável em separado, percebe-se uma variabilidade nas respostas no tocante às emoções negativas e positivas, com destaque para as variáveis orgulhoso ( $D P=1,391)$, empolgado ( $D P=1,247)$, preocupado $(D P=1,167)$ e incomodado ( $D P=1,182$ ). Ou seja, aparentemente, nem todos os funcionários ativos concordam que se sentem orgulhosos, empolgados ou que estejam preocupados e incomodados em trabalhar nesse hospital. A variável "atualmente no meu trabalho consigo recompensas importantes para mim" também apresentou o maior desvio padrão ( $D P=1,182)$ no fator realização no trabalho. Assim, também há evidências estatísticas de que, aparentemente, nem todos os funcionários do hospital percebem que conseguem ganhos e benefícios no trabalho.

Tabela 1 - Estatística descritiva dos profissionais ativos dos hospitais

\begin{tabular}{lcc} 
& Média & Desvio padrão \\
Realização & $\mathbf{4 , 2 3}$ & $\mathbf{0 , 5 9 6}$ \\
\hline Desenvolvo habilidades que considero importantes & 4,46 & 0,795 \\
\hline Expresso o que há de melhor em mim & 4,46 & 0,795 \\
\hline Faço o que realmente gosto de fazer & 4,45 & 0,859 \\
\hline Supero desafios & 4,40 & 0,818 \\
\hline Realizp atividades que expressem minhas capacidades & 4,34 & 0,881 \\
\hline Atinjo o resultados que valorizo & 4,19 & 0,866 \\
\hline Avanço nas metas que estabaleci para minha vida & 4,08 & 0,957 \\
\hline Realizo o meu potencial & 4,01 & 1,023 \\
\hline Consigo recompensas importantes para mim & 3,71 & 1,182 \\
\hline
\end{tabular}




\begin{tabular}{lll}
\hline Afeto positivo & $\mathbf{3 , 0 8}$ & $\mathbf{0 , 8 6 0}$ \\
\hline Disposto & 3,39 & 0,986 \\
\hline Feliz & 3,21 & 1,159 \\
\hline Contente & 3,20 & 0,963 \\
\hline Tranquilo & 3,18 & 1,158 \\
\hline Alegre & 3,14 & 0,923 \\
\hline Animado & 3,06 & 1,090 \\
\hline Orgulhoso & 2,94 & 1,391 \\
\hline Entusiasmado & 2,84 & 1,145 \\
\hline Empolgado & 2,77 & 1,247 \\
\hline Afeto negativo & $\mathbf{2 , 0 4}$ & $\mathbf{0 , 6 9 8}$ \\
\hline Preocupado & 2,93 & 1,167 \\
\hline Ansioso & 2,61 & 1,082 \\
\hline Irritado & 2,20 & 1,039 \\
\hline Chateado & 2,18 & 1,062 \\
\hline Tenso & 2,10 & 1,062 \\
\hline Incomodado & 1,99 & 1,182 \\
\hline Impaciente & 1,92 & 0,970 \\
\hline Frustrado & 1,88 & 1,126 \\
\hline Nervoso & 1,86 & 1,017 \\
\hline Entendiado & 1,70 & 0,914 \\
\hline Com raiva & 1,67 & 0,930 \\
\hline Deprimido & 1,44 & 0,822 \\
\hline Fonte Dados & & \\
\hline
\end{tabular}

Fonte: Dados da pesquisa; elaborado pelos autores.

Em particular, quanto às emoções, ao analisar a Tabela 2, pode ser constatado que a maioria desses funcionários tem a percepção de vivenciar no trabalho tanto emoções positivas e prazerosas quanto negativas e aflitivas. Pelo menos $60 \%$ dos funcionários ativos percebem que, no ambiente desse hospital, têm vivenciado emo- ções positivas e 70\% têm sentido pouco as emoções negativas. Ou seja, por um lado, cerca de $40 \%$ desses funcionários ativos têm sentido as emoções positivas muito pouco, por outro lado, cerca de $30 \%$ percebem que as emoções desagradáveis, aflitivas e angustiantes têm impactado bastante o bem-estar.

Tabela 2 - Comparação entre as emoções positivas e negativas dos profissionais ativos dos hospitais

\begin{tabular}{lccc} 
Afeto Positivo & $\begin{array}{c}\text { Nos últimos seis meses, } \\
\text { meu trabalho tem me } \\
\text { deixado moderadamente/ } \\
\text { bastante/extremamente... (\%) }\end{array}$ & $\begin{array}{c}\text { Nos últimos seis meses, } \\
\text { meu trabalho tem me } \\
\text { deixado nem um poucol } \\
\text { um pouco... (\%) }\end{array}$ \\
\hline Disposto & 84,1 & Deprimido & 96,2 \\
\hline Alegre & 78,5 & Entediado & 93,9 \\
\hline Contente & 78,8 & Com raiva & 93,9 \\
\hline
\end{tabular}




\begin{tabular}{lccc}
\hline Tranquilo & 75,8 & Impaciente & 91,7 \\
\hline Animado & 75,0 & Nervoso & 90,9 \\
\hline Feliz & 73,5 & Tenso & 87,9 \\
\hline Entusiasmado & 64,4 & Irritado & 87,9 \\
\hline Orgulhoso & 64,4 & Chateado & 86,4 \\
\hline Empolgado & 60,6 & Incomodado & 86,4 \\
\hline & & Ansioso & 77,3 \\
\hline & & Preocupado & 68,9 \\
\hline
\end{tabular}

Fonte: Dados da pesquisa; elaborada pelos autores.

\subsection{Estatística descritiva dos profissionais que saíram voluntariamente dos hospitais}

De acordo com a Tabela 3, a média do fator realização no trabalho $(M=3,83)$ mostra evidências estatísticas de que os funcionários que saíram voluntariamente concordam em parte que conseguem se realizar no trabalho. Mas, concordam que fazem o que realmente gostam de fazer $(\mathrm{M}=$ $4,14)$ e desenvolvem habilidades que consideram importantes $(M=4,23$. A média do fator afeto positivo $(M=3,20)$ mostra evidências estatísticas de que a afeição ao trabalho impactou de forma moderada o bem-estar dos funcionários que tomaram a decisão pela saída voluntária do hospital. Já a média do fator afeto negativo $(M=2,03)$ mostra evidências estatísticas de que emoções negativas quanto ao trabalho impactaram um pouco o bem-estar desses funcionários.

O desvio padrão para todos os fatores - realização ( $D P=0,709)$, afeto positivo (DP = 0,803) e afeto negativo ( $D P=0,676)$ - é similar e está abaixo de 1,0, mostrando pouca variabilidade nas respostas, o que indica coerência nas respostas dos funcionários que tomaram a decisão pela saída voluntária do hospital. Ou seja, as evidências estatísticas mostram que, aparentemente, houve coerência entre as situações e acontecimentos vivenciados por esses funcionários no tocante às emoções e às oportunidades de realização nesse ambiente organizacional.

Tabela 3 - Estatística descritiva dos profissionais que saíram voluntariamente da dos hospitais

\begin{tabular}{lcc} 
& Média & Desvio Padrão \\
\hline Realização & $\mathbf{3 , 8 3}$ & $\mathbf{0 , 7 0 9}$ \\
\hline Faço o que realmente gosto de fazer & 4,23 & 1,007 \\
\hline Eesenvolvo habilidades que considero importantes & 4,14 & 0,983 \\
\hline Supero desafios & 3,97 & 1,044 \\
\hline Atinjo resultados que valorizo & 9,36 & 1,011 \\
\hline Realizo atividades que expressam minhas capacidades & 3,85 & 0,981 \\
\hline Avanço nas metas que estabeleci para minha vida & 3,85 & 1,041 \\
\hline Realizo o meu potencial & 3,65 & 1,095 \\
\hline Consigo recompensas importantes para mim & 3,53 & 0,997 \\
\hline
\end{tabular}




\begin{tabular}{lll}
\hline Afeto positivo & $\mathbf{3 , 2 0}$ & $\mathbf{0 , 8 0 3}$ \\
\hline Disposto & 3,73 & 1,067 \\
\hline Animado & 3,35 & 1,088 \\
\hline Feliz & 3,34 & 1,147 \\
\hline Alegre & 3,32 & 0,972 \\
\hline Contente & 3,25 & 1,033 \\
\hline Tranquilo & 3,14 & 0,997 \\
\hline Entusiasmado & 3,10 & 1,079 \\
\hline Orgulhoso & 2,92 & 1,320 \\
\hline Empolgado & 2,72 & 1,117 \\
\hline Afeto negativo & $\mathbf{2 , 0 3}$ & $\mathbf{0 , 6 7 6}$ \\
\hline Preocupado & 2,85 & 1,125 \\
\hline Ansioso & 2,58 & 1,089 \\
\hline Irritado & 2,20 & 1,062 \\
\hline Tenso & 2,15 & 1,125 \\
\hline Chateado & 2,11 & 1,046 \\
\hline Incomodado & 2,05 & 1,127 \\
\hline Frustrado & 1,94 & 1,043 \\
\hline Impaciente & 1,84 & 0,936 \\
\hline Nervoso & 1,84 & 0,860 \\
\hline Com raiva & 1,71 & 0,958 \\
\hline Fon & & \\
\hline
\end{tabular}

Fonte: Dados da pesquisa; elaborada pelos autores.

Contudo, ao analisar cada variável separadamente, nesse grupo também se percebe uma variabilidade nas respostas no tocante às emoções negativas e positivas, com destaque também para a variável orgulhoso ( $D P=1,320)$, que também apresentou uma das menores médias ( $M=2,92)$, e preocupado ( $D P=1,125$ ). Ou seja, aparentemente, nem todos os funcionários que solicitaram demissão voluntária do hospital concordaram que se sentiram orgulhosos em trabalhar nesses hospitais ou que estivessem preocupados. A variável "atualmente no meu trabalho consigo recompensas importantes para mim" também apresentou o maior desvio padrão ( $D P=1,128)$ no fator realização no trabalho, que também apresentou a menor média $(M=3,71)$. Assim, nesse grupo, também há evi- dências estatísticas de que, aparentemente, nem todos os funcionários que tomaram a decisão pela saída voluntária do hospital perceberam que conseguiram recompensas no trabalho.

Quanto às emoções, ao analisar a Tabela 4 , pode ser constatado que pelo menos $55 \%$ dos funcionários que solicitaram demissão voluntária do hospital perceberam que no ambiente organizacional no qual estavam inseridos vivenciaram emoções positivas. Já no tocante às emoções negativas, pelo menos $70 \%$ dos funcionários sentiram pouco essas emoções e cerca de 30\% perceberam que as emoções desagradáveis, aflitivas e angustiantes impactaram bastante o bem-estar desses funcionários. Essas evidências se assemeIham às dos profissionais ativos. 
Tabela 4 - Comparação entre as emoções positivas e negativas dos profissionais que saíram voluntariamente da dos hospitais

\begin{tabular}{cccc} 
Afeto Positivo & $\begin{array}{c}\text { Nos últimos seis meses, meu } \\
\text { trabalho tem me deixado } \\
\text { moderadamente/bastante/ } \\
\text { extremamente... (\%) }\end{array}$ & Afeto Negativo & $\begin{array}{c}\text { Nos últimos seis meses, } \\
\text { meu trabalho tem me } \\
\text { deixado nem um pouco/um } \\
\text { pouco... (\%) }\end{array}$ \\
\hline Disposto & 85,0 & Deprimido & 97,7 \\
\hline Alegre & 78,8 & Entendiado & 94,0 \\
\hline Contente & 78,2 & Com raiva & 95,5 \\
\hline Animado & 76,0 & Impaciente & 93,2 \\
\hline Feliz & 73,7 & Frustrado & 93,2 \\
\hline Entusiasmado & 72,9 & Chateado & 90,2 \\
\hline Tranquilo & 69,2 & Tenso & 88,0 \\
\hline Orgulhoso & 62,4 & Incomodado & 87,2 \\
\hline Empolgado & 55,6 & Irritado & 86,5 \\
\hline & Ansioso & 85,7 \\
\hline & & Preocupado \\
\hline
\end{tabular}

Fonte: Dados da pesquisa; elaborada pelos autores.

\subsection{TESTE T ENTRE OS PROFISSIONAIS ATIVOS E OS PROFISSIONAIS QUE TOMARAM A DECISÃO PELA SAÍDA VOLUNTÁRIA}

Conforme mostrado na Tabela 5, para o fator realização no trabalho, há diferenças estatisticamente significativas $(p<0,05)$ entre os funcionários ativos e os que tomaram a decisão pela saída voluntária dos hospitais. Em outras palavras, as evidências mostram que os profissionais que ainda estão trabalhando no hospital estão significativamente mais realizados pessoalmente nesse trabalho do que aqueles que solicitaram a demissão voluntária do hospital. A única exceção nesse fator é a variável "atualmente no meu trabalho faço o que realmente gosto de fazer". Ou seja, não há evidências de diferenças estatisticamente significativas entre funcionários ativos e aqueles que tomaram a decisão pela saída voluntária.

Tabela 5 - Estatísticas de grupos entre os profissionais ativos e dos profissionais que tomaram decisão de demissão voluntária dos hospitais

\begin{tabular}{lllll}
\hline \multirow{2}{*}{ Realização* $^{*}$} & N & Média & Desvio padrão \\
\hline \multirow{2}{*}{ Faço o que realmente gosto de fazer** } & Ativo & 132 & 4,23 & 0,597 \\
& Demissionário & 133 & 3,83 & 0,710 \\
\hline \multirow{2}{*}{ Afeto positivo* } & Ativo & 132 & 4,45 & 0,859 \\
& Demissionário & 133 & 4,23 & 1,007 \\
\hline \multirow{2}{*}{ Disposto* } & Ativo & 132 & 3,08 & 0,860 \\
& Demissionário & 133 & 3,20 & 0,803 \\
\hline \multirow{2}{*}{ Animado* } & Ativo & 132 & 3,39 & 0,986 \\
& Demissionário & 133 & 3,73 & 1,067 \\
\hline \multirow{2}{*}{ Afeto negativo* } & Ativo & 132 & 3,06 & 1,090 \\
& Demissionário & 133 & 3,35 & 1,088 \\
\hline
\end{tabular}

Fonte: Dados da pesquisa; elaborado pelos autores. ${ }^{*}(p<0,05)$. 
Quanto ao fator afeto positivo, embora não haja evidências de diferenças estatísticas ( $p$ $<0,05)$, duas variáveis que compõem esse fator - disposição e animação - apresentam diferenças estatísticas. Nesse caso, são os funcionários que solicitaram a demissão voluntária do hospital que estão significativamente mais dispostos e animados com o trabalho nesses hospitais. Já o fator afeto negativo não apresentou evidências de diferenças estatisticamente significantes $(p<0,05)$.

\section{DISCUSSÃO DOS RESULTADOS}

Passa-se agora a comparar, a partir dos fatores situacionais e pessoais identificados, os impactos sobre o bem-estar no trabalho dos profissionais ativos e de profissionais que decidiram pela demissão voluntária de uma organização de grande porte no segmento da saúde na Grande Vitória.

Os resultados mostraram evidências estatísticas de que esse ambiente organizacional de prestação de serviços de saúde, tanto para a maioria dos funcionários que ainda trabalham nesses hospitais como para a maioria daqueles que tomaram a decisão voluntária de sair, tem despertado ou despertou emoções positivas com destaque para alegria, disposição, contentamento, animação, felicidade. Curiosamente, as emoções entusiasmo, orgulho e empolgação, para ambos os grupos, são as menos sentidas, já que a profissão selecionada conta com uma especificidade, que é o cuidado com a vida (BACCARO; SHINYASHIKI, 2011). Chama a atenção que os funcionários que solicitaram demissão voluntária se sentiram mais dispostos e animados nesse ambiente de trabalho do que os ativos. Talvez pelo fato de estarem vivenciando no- vas oportunidades e projetos, uma vez que a survey "Entrevista de Desligamento" mostrou que a maioria dos respondentes disse que o motivo da saída era mudança de emprego.

Quanto aos sentimentos negativos, os funcionários percebem ou perceberam que esse ambiente organizacional de prestação de serviços de saúde tem despertado ou despertou só um pouco emoções como depressão, tédio, raiva, nervosismo e impaciência, não havendo diversidade de entendimento entre os dois grupos. Ou seja, embora esse setor seja notoriamente conhecido por ser um ambiente que provoca altos níveis de tensão, esgotamento físico e mental e estresse, denominados Síndrome de Burnout (JODAS; HADDAD, 2009; SILVA, 2011), a maioria dos funcionários ativos e dos profissionais que solicitaram demissão voluntária percebem/perceberam que o trabalho nesse hospital tem despertado ou despertou mais emoções de afeição ao trabalho do que negativas. Bradbrun (1969) já mencionava que algumas pessoas, no ambiente de trabalho, são mais felizes do que outras, devido à vivência de experiências mais positivas do que negativas. Isto não significa que elas não tenham vivenciado condições negativas. Esses resultados também corroboram os achados de vários pesquisadores (SANT'ANNA; PASCHOAL; GOSENDO, 2012; PASCHOAL; TAMAYO, 2008), de que as emoções positivas e negativas não são excludentes e fazem parte do convívio diário no trabalho, independentemente do setor de atuação.

Para os funcionários ativos, essas emoções podem ser resultado da percepção de realização nesse trabalho, uma vez que pelo menos $70 \%$ desses funcionários disseram que conseguem: realizar todo o potencial, desenvolver habilidades 
que consideram importantes, realizar atividades que expressam suas capacidades, superar desafios (PAZ; DESSEN, 2010), cumprir os objetivos traçados e expressar o que há de melhor neles. Isso pode ser devido ao fato de também fazerem o que realmente gostam. Como resultado, a maioria desses funcionários percebem que conseguem avançar nas metas que estabeleceram para suas vidas. Contudo, cabe destacar que nesse grupo 30\% discorda dessas realizações, mostrando que há necessidade de oportunidades de melhorias nesse hospital no tocante à gestão de pessoas com o intuito de meIhorar o bem-estar dos funcionários.

Já para o grupo de profissionais que solicitaram a demissão voluntária, somente a metade conseguiu realizar atividades que expressam: i) Capacidades, ii) superaram desafios (PAZ; DESSEN, 2010), iii) cumprem os objetivos traçados e iv) expressam o que há de melhor neles. Mas sobe para $60 \%$ aqueles que perceberam que conseguiram desenvolver habilidades consideradas importantes e avançar nas metas que estabeleceram para suas vidas. Isso também pode ser devido, em parte, ao fato de que esses funcionários fizeram o que realmente gostam, já que menos de $40 \%$ perceberam que nesse hospital conseguiram realizar o potencial e fazer o que realmente gostam de fazer. Essa percepção pode ser devido às evidências de não haver diferenças entre a percepção quanto ao fazer o que realmente gosta entre os dois grupos. Novamente, isso pode ocorrer por conta da especificidade da profissão, que é o cuidado com a vida (BACCARO; SHINYASHIKI, 2011). Esses achados corroboram com os estudos de Paschoal, Torres e Porto (2010), em que resultados semelhantes são explicados por variáveis que influenciam os afetos positivos, ou seja, quão maior for o bem-estar positivo, maior a probabilidade de se conseguir desenvolver as metas organizacionais estabelecidas. Embora Ferreira e Almeida (2015) sugerem que quanto maior o investimento em treinamentos maior a taxa de rotatividade.

Para ambos os grupos, ganhar recompensas importantes é a variável menos percebida. Poeira e Mamede (2011) também defendem que os fatores contratuais e remuneratórios são relevantes na medida em que o funcionário se sente sensível devido à instabilidade contratual e à baixa remuneração. As saídas voluntárias podem estar ocorrendo por outras variáveis que não seja recompensa, como estudado por Pilla e Sanches (2008), como escassez de recursos e condições de trabalho inapropriadas. Ou por estratégias na contramão da gestão, infelicidade e baixa autoestima do funcionário da área da saúde (MENDES; MARZIALE, 2006), aliados à assistência insuficiente e de qualidade muito questionada, além dos altos índices de tensão na organização (PASCHOALINI et al., 2008). Ou seja, há "necessidade da implantação de práticas organizacionais que representem uma retribuição compatível com os esforços individuais" (SANT'ANNA; PASCHOAL; GOSENDO, 2012, p. 760). Portanto, os resultados acima evidenciam que a maior parte dos funcionários vem estreitando a necessidade dos aspectos afetivos com a organização (PASCHOAL; TAMAYO, 2008). Logo, os hospitais deveriam adotar um relacionamento com base na troca social, ou seja, na reciprocidade, que por sua vez depende da confiança e enfatiza os fatores socio-emocionais da relação com estes funcionários, e não somente na troca econômica, que enfatiza os aspectos financeiros (MIHAIL; KLOUTSINIOTIS, 2016). 


\section{CONSIDERAÇÕES FINAIS}

Os resultados mostram evidências de que, no ambiente organizacional de prestação de serviços de saúde estudada, sentimentos de afeto positivo e negativo e de realização no trabalho podem influenciar em maior ou menor grau o bem-estar, tanto dos funcionários que ainda trabalham nesse hospital como daqueles que tomaram a decisão voluntária de sair. No tocante à realização do potencial no trabalho e às competências individuais, os funcionários ativos concordam que conseguem desenvolver e aplicar mais o conhecimento, as habilidades e atitudes no dia a dia dos hospitais. Como resultado, conseguem cumprir metas pessoais, organizacionais e, como retorno, receber recompensas consideradas importantes. Já os funcionários que solicitaram a demissão voluntária do hospital tiveram uma percepção um pouco menor e apenas concordam em parte com essa percepção.

Por isso, aparentemente, os sentimentos positivos e negativos e de realização podem não ter sido as principais razões para a tomada de decisão de saída voluntária desse hospital. Contudo, no tocante à obtenção de recompensas importantes, está em consonância com os resultados da survey feita no momento de desligamento dos profissionais, que apontou remuneração e benefícios como algumas das principais razões para a saída da organização. Tamanha relevância do construto recompensa na amostra é perceptível, que existe insatisfação pelo motivo da falta ou ausência de recompensa, nas demonstrações de sentimentos nas relações de trabalho, dentro do ambiente organizacional. Desta forma, cabe a essa organização investigar outras possíveis causas da rotatividade, uma vez que $77 \%$ dos respondentes - ativos e profissionais que solicitaram demissão voluntária - têm ou estavam com até dois anos de casa.

Um grande desafio nas organizações é buscar atratividades na recompensa de seus funcionários visando a retenção de seus talentos, uma vez que o alto índice de rotatividade e uma amostra extensa de funcionários novos interferem diretamente nos resultados de perspectiva de entrega de qualidade dos serviços aos clientes, além do impacto econômico financeiro. Outro ponto importante é contribuir com a equipe para que possa entregar em nível ótimo a realização de seu potencial, outro motivo pelo qual a recompensa auxilia nessa relação. Porém, recompensa não é um fator isolado. A liderança, que não é ação de governabilidade do setor de RH e sim dos próprios líderes, foi identificada como fragilidade na decisão das saídas registradas na resposta da survey. Essa é outra questão que deve ser trabalhada, pois pode estar influenciando a tomada de decisão nas saídas. Ou seja, desenvolvimento e retenção de lideranças para enfrentar e decidir nas situações do cotidiano são essenciais para uma organização mais saudável.

Esse estudo tem limitações, tais como o tamanho da amostra e a impossibilidade de generalização dos resultados. O preenchimento do questionário foi solicitado aos funcionários ativos e aplicados aos funcionários que solicitaram demissão voluntária diretamente pela Diretora de um dos hospitais, o que pode ter contribuído para a inibição e rejeição dos funcionários quanto à participação na pesquisa, notadamente no caso de funcionários ativos em que foi obtido um baixo índice de respostas. Além disso, trata-se de uma amostra não probabilística, por acessibilidade. 
Apesar dessas limitações, esse estudo contribui com uma pesquisa empírica para a área de gestão de pessoas ao discutir a percepção de bem-estar entre os profissionais ativos e demissionários, uma vez que não se verifica, especialmente no Brasil, estudos abordando bem-estar e estes dois grupos. Contribui, em particular, no avanço das generalizações ao comparar esses dois grupos dentro da mesma organização, em outro segmento do mercado (segmento da saúde), cidade e região (Região Metropolitana da Grande Vitória). E, em termos práticos, mostra os benefícios da Escala de Bem-Estar no Trabalho (EBET), de Paschoal e Tamayo (2008), recomendando-a a sua inserção na rotina de gestão de pessoas nas organizações para auxiliar, por exemplo, na análise anual de seus funcionários, objetivando avaliar o nível de bem-estar e felicidade do patrimônio humano da organização, bem como tratar suas fraquezas, buscando assim um posicionamento competitivo no mercado. Como sugestões futuras, outros eixos de discussão que permeiam o bem-estar, como recompensa e realização do potencial do indivíduo nas organizações, são temas de relevância teórica. Outra sugestão é aplicar esse estudo associado a duas variáveis simultâneas como absenteísmo ocupacional e não ocupacional e rotatividade voluntária, recompensas, além de aplicar a variável clima organizacional, bem como de estender o estudo para outros segmentos de mercado.

\section{REFERÊNCIAS}

AGAPITO, P. R.; POLIZZI FILHO, A.; SIQUEIRA, M. M. M. Bem-estar no trabalho e percepção de sucesso na carreira como antecedentes de intenção de rotatividade. RAM, Revista de Administração Mackenzie, v. 16, n. 6, Edição Especial, 2015.

ALBUQUERQUE, A. S. TRÓCCOLI, B. T. Desenvolvimento de uma escala de bem-estar subjetivo. Psicologia: Teoria e Pesquisa, v. 20, n. 2, p.154164, 2004.

ASHKANASY, N. M. Thirty years of shaping a discipline: JOB's most influential papers. Journal of Organizational Behavior, v. 30, n. 8 p. 1019 $-1024,2009$.

ASSIS, M. T. Indicadores de gestão de recursos humanos: usando indicadores demográficos, fi- nanceiros e de processos na gestão de capital humano. Rio de Janeiro: Qualitymark Editora, 2012.

BACCARO, T. A.; SHINYASHIKI, G. T. Consistência da escolha vocacional e socialização profissional de estudantes de enfermagem. Revista Brasileira de Orientação Profissional, v. 12, n.1, p. 73$82,2011$.

BARBIERI, J. C.; VASCONCELOS, I. F. G.; ANDREASSI, T.; VASCONCELOS, F. C. Inovação e sustentabilidade: novos modelos e proposições 2010.

RAE Revista de Administração de Empresas, v. 50, n. 2, p. 146-154, 2010.

BARCAUI, A.; FRANÇA, A. C. L. Estresse, enfrentamento e qualidade de vida: um estudo sobre gerentes brasileiros. Revista de Administração Contemporânea, v. 18, n. 5, p. 670-694, 2014. 


\section{REFERÊNCIAS}

BRAGA, L. T.; ANDRADE, T.; ESTIVALETE, V. F. B.; OLIVEIRA, J. M.; COSTA, V. F. Valores Organizacionais: Preditores do Bem-Estar no Trabalho? Gestão \& Regionalidade, v. 33, n. 99, p. 170-187, 2017.

BEHSON, S. J. The relative contribution of formal and informal organizational work family support. Journal of Vocational Behavior, v. 66, n. 3, p. 487-500, 2005.

BENSON, J.; BROWN, M. Generations at work: are there differences and do they matter? The International Journal of Human Resource Management, v. 22, n. 9, p. 1843-1865, 2011.

BERNSTORFF, V. H. Relações entre satisfação, competência, saúde e absenteísmo no trabalho em uma grande instituição bancária pública. Sociedade e Estado, v. 23, n. 1, p. 189-190, 2008. CAMPOS, C. V. A.; MALIK, A. M. Satisfação no trabalho e rotatividade dos médicos do Programa de Saúde da Família. Revista de Administração Pública, v. 42, n. 2, p. 347-368, 2008.

Chen, L., Gong, T., Kosinski, M., Stillwell, D., \& Davidson, R. L. Building a profile of subjective well-being for social media users. PloS one, v. 12, n. 11, p. e0187278, 2017.

DIENER, E. Subjective well-being: The science of happiness and a proposal for a national index. American Psychologist, v. 55, n. 1, p. 34-43, 2000.

DIENER, E.; HORWITZ, J.; EMMONS, R.A. Happiness of the very wealthy. Social Indicators Research, n. 16, p. 263-274, 1985.
DIENER, E. The remarkable changes in the science of subjective well-being. Perspectives on Psychological Science, v. 8, n. 6, p. 663-666, 2013.

Diener, E.; Suh, E. Measuring Quality Of Life: Economic, Social and Subjective Indicators. Social Indicators Research, v. 40, p. 189-216, 1997.

DUFFY, R. D.; BOTT, E. M.; ALLAN, B. A; AUTIN, K. L. Calling among the unemployed: Examining prevalence and links to coping with job loss. Journal of Positive Psychology, v. 10, n. 4, p. 332-345. 2015.

ELIAS, M. A.; NAVARRO, V. L. A relação entre o trabalho, a saúde e as condições de vida: negatividade e positividade no trabalho das profissionais de enfermagem de um hospital escola. Revista Latino-Americana Enfermagem, v. 14, n. 4, p. 517-25, 2006.

Fan, D., Cui, L., Zhang, M. M., Zhu, C. J., Härtel, C. E., \& Nyland, C. Influence of high performance work systems on employee subjective well-being and job burnout: empirical evidence from the Chinese healthcare sector. The International Journal of Human Resource Management, v. 25, n. 7, p. 931-950, 2014.

FERREIRA, L. C. M.; ALMEIDA, C. B. A. BBR, Brazilian Business. Review, v. 12, n. 4, p. 28 - 61, 2015.

Fisher, C. D. Happiness at Work. International journal of management reviews, v. 12, n. 4, p. 384-412, 2010.

FREDRICKSON, B. L. The Value of Positive Emotions. American Scientist, v. 91, p. 330335, 1998. 


\section{REFERÊNCIAS}

FREIRE, P. Pedagogia da Autonomia. Rio de Janeiro: Paz e Terra, 1997.

GOETZ, K.; BERGER, S.; GAVARTINA, A.; ZAROTI, S.; SZECSENYI, J. How psychosocial factors affect well-being of practice assistants at work in general medical care?-a questionnaire survey. BMC family practice, v. 16, n. 1, p. 166, 2015.

HAASE, V. G., O desenvolvimento humano como busca da felicidade. In: HAASE, V. G.; FERREIRA, F. O.; PENNA, F. J. Aspectos biopsicossociais da saúde na infância e adolescência, Publisher: Belo Horizonte: 2001. p.601-635.

Hair Jr., J. F. et. al. Fundamentos de métodos de pesquisa em administração. Porto Alegre: Bookman, 2005.

HEFFERNAN, M; DUNDON, T. Cross-level effects of high-performance work systems (HPWS) and employee well-being: the mediating effect of organisational justice. Human Resource Management Journal, v. 26, n. 2, p. 211-231, 2016.

HOUAISS, A.; VILLAR, M. S.; MELLO FRANCO, F. $M$. Dicionário Houaiss da Língua Portuguesa. Rio de Janeiro: Objetiva, 2009.

JODAS, D. A.; HADDAD, M. C. L. Síndrome de Burnout em trabalhadores de enfermagem de um pronto socorro de hospital universitário. Acta Paulista de Enfermagem, v. 22, n. 2, p. 192- 197, 2009.

KNUDSEN, H. K.; DUCHARME, L. J.; ROMAN, P. M. Clinical supervision, emotional exhaustion, and turnover intention: A study of substance abuse treatment counselors in the Clinical Trials Network of the National Institute on Drug Abuse. Journal of substance abuse treatment, v. 35, n. 4, p. 387-395, 2008.

LACHOWSKA, Marta. The Effect of Income on Subjective Well-Being: Evidence from the 2008 Economic Stimulus Tax Rebates. Journal of Human Resources, 2016.

LEE H. et al. Predictors of life satisfaction of Korean nurses. Journal of Advanced Nursing, v. 48, n. 6, p. 632-643, 2004.

LOCKE, E. A. The nature and causes of job satisfaction. In: DUNNETTE, M. P. (Org.), Handbook of Industrial and Organizational Psychology, p. 1294-1349. Chicago: Rand-McNally, 1976.

LOPES, M. J. M.; LEAL, S. M. C. A feminização persistente na qualificação profissional da enfermagem brasileira. Cadernos Pagu, n. 24, p.105-125, 2005.

LYUBOMIRSKY, S.; KING, L.; DIENER, E. The benefits of frequent positive affect: does happiness lead to success? Psychological Bulletin, v. 131, n.6, p. 803-855, 2005.

MALHOTRA, N. K. Pesquisa de marketing: uma orientação aplicada. 4. ed. Porto Alegre: Bookman, 2006.

MENDES, C. A. I.; MARZIALE, P. H. M. Década de Recursos Humanos em Saúde: 2006-2015. Revista Latino-Americana Enfermagem, v. 14, n.1, p. 1-2, 2006. 


\section{REFERÊNCIAS}

MELLOR, David; MOORE, Kathleen A.; SIONG, Zhong Ming Benjamin. The role of general and specific stressors in the health and well-being of call centre operators. Work, v. 52, n. 1, p. 31-43, 2015.

MIHAIL, D. M.; KLOUTSINIOTIS, P. V. The effects of high-performance work systems on hospital employees' work-related well-being: Evidence from Greece. European Management Journal, v. 34, n. 4, p. 424-438, 2016.

NARDI, C. F. F.; PALMA, D. L Bem-estar no trabalho: contribuições à prática social nas empresas. Revista Tecnológica, v. 2, n. 1, 2015.

NOMURA, F. H.; GAIDZINSKI, R. R. Rotatividade da equipe de enfermagem: estudo em hospital-escola. Revista Latino-Americana Enfermagem, v. 13, n.5. 2005.

PAZ, T. G. M.; DESSEN, G. M. Validação do instrumento de indicadores de bem-estar pessoal nas organizações. Psicologia em Estudo, v. 15, n. 2, p. 409-418, 2010.

PASCHOAL, T.; TAMAYO, A. Construção e validação da escala de bem-estar no trabalho. Avaliação Psicológica, v. 7, n. 1, p. 11-22, 2008.

PASCHOAL, T.; TORRES, C. V.; PORTO, J. B. Felicidade no trabalho: relações com suporte organizacional e suporte social. Revista de Administração Contemporânea (RAC), v.14, n. 6, p.1054-1072, 2010.

PASCHOALINI, B.; OLIVEIRA, M. M.; FRIGERIO, M. C.; DIAS, A. L. R. P.; SANTOS, F. H. Cognitive and emotional effects of occupational stress in nursing professionals. Acta Paulista de Enfermagem, v. 21, n.3, p. 487-492, 2008.

POEIRA, A.; MAMEDE, R. P. Os fatores determinantes da rotatividade externa dos enfermeiros: vínculo contratual, incentivos salariais ou reconhecimento profissional. Revista de Enfermagem, SER III, n. 4, p. 107-114, 2011.

RAHIM, Norizan Baba. Leveraging the Psychological Well-Being among Malaysian Engineers: The Role of Protean Career Orientation and Career Strategy Implementation. Jurnal Pengurusan (UKM Journal of Management), v. 49, 2017.

REEVE, J.; DECI, E. L. Elements of the competitive situation that affect intrinsic motivation. Personality and Social Psychology Bulletin, n. 22, , n.1, p. 24-33, 1996.

REGO, A.; E CUNHA, M. P. They need to be different, they feel happier in authentizotic climates. Journal of Happiness Studies, v. 13, n. 4, p. 701-727, 2012.

REGO A. Funcionários felizes são mais produtivos? Revista dos Estudos Politécnicos. v. 7, n. 12, p. 215-233, 2009.

Rickard, G., Lenthall, S., Dollard, M., Opie, T., Knight, S., Dunn, S. Organisational intervention to reduce occupational stress and turnover in hospital nurses in the Northern Territory, Australia. Collegian, v. 19, n. 4, p. 211-221, 2012. 


\section{REFERÊNCIAS}

SANCHO, L. G.; CARMO, J. M.; SANCHO, R. G.; BAHIA, L. Rotatividade na força de trabalho da rede municipal de saúde de Belo Horizonte, Minas Gerais: um estudo de caso. Trabalho, Educação e Saúde, v. 9, n. 3, 2011.

SANT'ANNA, L. L.; PASCHOAL, T.; GOSENDO, E. E. M. Bem-estar no trabalho: relação com estilo de liderança e suporte para ascensão, promoção e salários. RAC. Revista de Administração Contemporânea, v. 16, n. 5, p. 744-764, 2012.

SCHIRRMEISTER, R.; LIMONGI-FRANÇA, A. C. A qualidade de vida no trabalho: relações com o comprometimento organizacional nas equipes multicontratuais. Revista Psicologia Organizações e Trabalho, v. 12, n. 3, p. 283-298, 2012.

SILVA, R. P.; BARBOSA, S. C.; SILVA, S. S.; PATRÍCIO, $D$. F. Burnout e estratégias de enfrentamento em profissionais de enfermagem. Arquivos Brasileiros de Psicologia, v. 67 n.1, 2015.

SIQUEIRA, M. M. M.; GOMIDE JR., S. Vínculos do indivíduo com o trabalho e com a organização. In: ZANELLI, J. C.; BORGES-ANDRADE, J. E.; BASTOS, A. V. B. (Orgs). Psicologia Organizações e Trabalho no Brasil. Porto Alegre: Artmed, 2014. p. 316-348.

SIQUEIRA, M. M. M.; PADOVAM, V. A. R. Bases teóricas de bem-estar subjetivo, bem-estar psicológico e bem-estar no trabalho. Psicologia: Teoria e Pesquisa, Brasília, v. 24, n. 2, p. 201-209, 2008.

SIU, O. L.; CHEUNG, F.; LUI, S. Linking positive emotions to work well-being and turnover intention among Hong Kong police officers: The role of psychological capital. Journal of happiness studies, v. 16, n. 2, p. 367-380, 2015.

SORAGGI, F.; PASCHOAL, T. Relação entre bem-estar no trabalho, valores pessoais e oportunidades de alcance de valores pessoais no trabalho. Estudos e Pesquisas em Psicologia, v.11, n. 2, p. 2011.

TORRISI, Benedetto. Academic productivity correlated with well-being at work. Scientometrics, v. 94, n. 2, p. 801-815, 2013.

VASCONCELOS, K. C. A.; MERHI, D. Q.; GOULART, V. M.; SILVA, A. R. L; A Geração Y e suas Âncoras de Carreira. GESTÃO.Org - Revista Eletrônica de Gestão Organizacional. V. 8, n. 2, p. 226-244, 2010.

VAN DEN BOSCH, R; TARIS, T. W. The authentic worker's well-being and performance: The relationship between authenticity at work, well-being, and work outcomes. The Journal of psychology, v. 148, n. 6, p. 659-681, 2014.

VAN DE VOORDE, K.; PAAUWE, J; VAN VELDHOVEN, $M$. Employee well-being and the HRM-organizational performance relationship: a review of quantitative studies. International Journal of Management Reviews, v. 14, n. 4, p. 391-407, 2012.

WARR, P. Learning about employee happiness. Revista Psicologia Organizações e Trabalho, v.7, n. 2, p.133-140, 2007.

WATERMAN, A. S; SCHWARTZ, S. J; CONTI, R. The implications of two conceptions of happiness (hedonic enjoyment and eudaimonia) for the understanding of intrinsic motivation. Journal of Happiness Studies. v. 9, n.1, p. 41-79, 2008. 